\title{
Assessing patient-caregiver communication in cancer-a psychometric validation of the Cancer Communication Assessment Tool (CCAT-PF) in a German sample
}

\author{
Markus W. Haun • Halina Sklenarova • Eva C. Winkler • Johannes Huber • \\ Michael Thomas • Laura A. Siminoff • Michael Woll • Anette Brechtel • \\ Wolfgang Herzog • Mechthild Hartmann
}

Received: 14 November 2013 / Accepted: 31 March 2014 / Published online: 11 April 2014

(C) Springer-Verlag Berlin Heidelberg 2014

\begin{abstract}
Purpose The recently introduced Cancer Communication Assessment Tool (CCAT-PF) measures congruence in patientcaregiver communication and was initially validated in lung cancer patients. Contributing to a greater proportion of the
\end{abstract}

M. W. Haun $(\bowtie) \cdot$ H. Sklenarova $\cdot$ A. Brechtel $\cdot$ W. Herzog $\cdot$ M. Hartmann

Department of General Internal Medicine and Psychosomatics, Heidelberg University Hospital, Im Neuenheimer Feld 410,

69120 Heidelberg, Germany

e-mail: markus.haun@med.uni-heidelberg.de

M. W. Haun

Soteria Bern, University Hospital of Psychiatry, Bern, Switzerland

E. C. Winkler

Program for Ethics and Patient-Oriented Care, National Centre for Tumour Diseases, Heidelberg University Hospital, Heidelberg,

Germany

J. Huber

Department of Urology, Heidelberg University Hospital, Heidelberg, Germany

M. Thomas

Department of Oncology, Thoraxklinik Heidelberg, Heidelberg

University Hospital, Heidelberg, Germany

\section{A. Siminoff}

Department of Social and Behavioral Health, School of Medicine,

Virginia Commonwealth University, Richmond, VA, USA

M. Woll

Medical School, Heidelberg University, Heidelberg, Germany

A. Brechtel

Division of Psycho-Oncology, National Centre for Tumour Diseases, Heidelberg University Hospital, Heidelberg, Germany variance in the conflict scores, primary caregivers were hypothesized to experience greater stress. For a detailed understanding of conflicting communication patterns of canceraffected families, our study aimed for psychometric validation of the CCAT-PF in a sample covering heterogeneous tumor entities.

Methods Subsequent to a cross-sectional survey of 189 pairs of cancer patients ( $31 \%$ gastrointestinal, $34 \%$ lung, and $35 \%$ urological) and their caregivers' exploratory factor analysis with principal component condensation and varimax rotation was conducted (response rate, $74.2 \%$ ). Reliability and construct validity were assessed calculating Cronbach's $\alpha$ and Pearson correlation coefficients for CCAT-P and CCAT-F scales and related constructs, respectively.

Results Cancer-related communication according to the CCAT-PF can be subdivided into four factors including the scales Disclosure, Limitation of treatment, Family involvement in treatment decisions, and Continuing treatment. Reliability ranged from $\alpha=.51-.68$. The Disclosure scale, describing poor cancer-related communication of the patient, was correlated with patient's distress (QSC-R10: $r=.30, p<.0001$ ), unmet needs in several areas (SCNS-SF-34: $r=.25-.32$, $p<.001$ ), and negatively with social/family well-being (FACT: $r=-0.31, p<.0001$ ). Higher scores on the scale were significantly associated with considerable decrements in emotional well-being especially for caregivers perceiving patients' disclosure as problematic.

Conclusions The Disclosure scale originating from the CCAT-PF emerged as a short, valid, and reliable stand-alone instrument for identifying conflicting communication in patient-caregiver-dyads at risk.

Keywords Cancer - Communication - Caregiver . Questionnaire · Validation · Distress 


\section{Introduction}

There is wide agreement that cancer may impact an entire family causing "fears, uncertainty, disrupted life plans, rearranged schedules and routines, changed interpersonal communication, existential worries, alterations in household members' functioning, and heightened household tension" [1, 2]. For example, Burns and colleagues identified fluctuating awareness of treatment goals among 163 cancer patients and their principal family caregivers [3]. It was figured that discrepancies in patient-caregiver-dyads might complicate the delivery of effective care at the latest when patients are seriously ill. However, valid and reliable instruments to assess types and quality of conflict in communication to specify key aspects for psychosocial interventions remain scarce. Recently, the Cancer Communication Assessment Tool for Patients and Families (CCAT-PF) was introduced as a measure of congruence in patient-family caregiver communication [4]. Construction was aimed at finding a clinical screening tool for family discord in cancer. Validation was conducted with a sample of 190 advanced stage non-small cell lung cancer patients and their primary caregivers [5]. The final scale comprised 18 items within eight domains, "a high sum total of the 18-item absolute difference scores indicating disagreement between patient and caregiver" [4]. Significantly, higher CCAT-PF conflict scores were exhibited by younger caregivers, adult children caregivers, and patients with lower income. Different domains of disagreement did not correlate with each other, underscoring the importance of separate examination of domain scores in addition to the CCAT-PF total score. As a greater proportion of the variance in the conflict scores that was contributed by caregivers, it was concluded that family caregivers are more aware of deviations from an open dialogue. Caregivers may therefore be experiencing greater stress. For a detailed understanding of conflicting communication patterns of cancer-affected families, our study aimed for German translation and psychometric reassessment of the CCAT-PF in a sample covering heterogeneous tumor entities.

\section{Methods}

Study design and sample Initial sample size calculation indicated that 134 subjects were necessary to test for sufficient reliability (Cronbach's $\alpha=.80$ ) [6]. Psychometric validation was conducted in a cross-sectional survey of 189 pairs of patients with diverse malignancies (31\% gastrointestinal, $34 \%$ lung, and $35 \%$ urological) and their primary caregivers. The patient sample was treated either as out- or inpatients at the National Centre for Tumour Diseases and the affiliated Thoraxklinik in Heidelberg. Caregivers, defined as nearest lay care providers, were determined by patients themselves, as in the initial study that developed the CCAT-PF. Data was collected on July/August 2012. Sociodemographic data was provided by patients and medical data was obtained from medical records and the attending physician. The study was approved by the Ethical Commission of Heidelberg Medical School (Ethic Approval/Registration Number: S-287/2012).

Measurement The German version of the CCAT-PF was based on a back translation by two independent translators. As there is no nationwide hospice care established in Germany and since most people are still unfamiliar with the idea [7], we excluded item 18 ("I would feel uncomfortable if the doctor began to talk to me about hospice care.") from the German version of the CCAT-PF. Furthermore, to assess construct validity, the questionnaire set comprised instruments assessing disease-related distress (Questionnaire on Distress in Cancer Patients-Short Form, QSC-R10) [8], depression and anxiety (Patient Health Questionnaire-4, PHQ-4) [9], and unmet needs (Supportive Care Needs Survey, SCNS-SF34/SCNS-P\&C-G) for patients and caregivers [10]. Additionally, patients completed the Functional Assessment of Cancer Therapy (FACTG/FACT-L for lung cancer patients respectively) [11, 12], while caregivers completed the Caregiver Strain Index (CSI) [13].

Statistical analysis Only complete pairs of questionnaire sets were included in the analyses. Four sets in which $>40 \%$ of CCAT-P and/or CCAT-F items were missing were excluded from the analysis. Descriptive statistics was computed for sample characteristics and item analysis. Evaluation of scale structure exploratory factor analysis with principal component condensation and varimax rotation was conducted [14]. The number of factors to be retained was determined according to Kaiser-Guttman criteria and the scree plot criteria. Assessment of reliability included estimation of internal consistency (Cronbach's $\alpha$ ). Construct validity was investigated using Pearson product-moment correlations. To identify differences between subgroups, the Student's $t$ tests and analyses of variance (ANOVA) were used. Non-responder analysis was conducted to test for representativeness of the sample regarding gender, age, and tumor type.

\section{Results}

Sociodemographic and medical data Three hundred thirtyone patients were screened for eligibility, 64 of whom were not eligible (no relative/caregiver present, $n=20$; insufficient German language proficiency, $n=8$; too recently diagnosed, $n=5$; poor general condition; assessed by attending physician, $n=2$; patient subjectively too distressed, $n=29$ ). Most of the patients who declared themselves as too distressed for 
participation were feeling too weak after high-dose chemotherapy treatment earlier on the same day. From the remaining 267 pairs, 44 declined participation and 25 did not return one of the two questionnaire sets (response rate, $74.2 \%$ ). Subjects refusing participation or not returning questionnaires did not differ significantly with respect to age $(p=.60)$ or gender $(p=.66)$ from those who did. However, lung cancer patients were more likely to refuse participation than subjects with urological or gastrointestinal tumors $(p<.001)$. Enrolled patients were predominantly male (68\%), had a mean age of 63 years, and had a post-secondary education level (56\%).
The majority was suffering from late-stage (metastasized) disease $(75 \%)$, had undergone chemotherapy in the last 2 months before the survey (78 \%), and had a WHO-ECOG performance status below 2 when assessed (91\%). Caregivers were predominantly female $(73 \%)$ and most commonly spouses $(84 \%)$, followed by adult children (12\%). Mean age of caregivers was 58 years with $57 \%$ of them having a post-secondary education level.

Item analysis and evaluation of the scale structure Extreme skew distribution ( $\mathrm{g}=2.86$ for the CCAT-P and 2.98 for

Table 1 Scale and item characteristics for CCAT-P and CCAT-F questionnaires

\begin{tabular}{|c|c|c|c|c|c|c|}
\hline \multirow[b]{2}{*}{ Scale 1} & \multirow[b]{2}{*}{ Disclosure } & \multirow[b]{2}{*}{ Factor loading } & \multicolumn{2}{|c|}{ CCAT-P } & \multicolumn{2}{|c|}{ CCAT-F } \\
\hline & & & Mean & SD & Mean & SD \\
\hline & Explained variance, $21.04 \%$ & - & 2.00 & .87 & 1.96 & .86 \\
\hline 2 & I hesitate to mention treatment side effects to my doctors or nurses. & .53 & 1.84 & 1.27 & 1.98 & 1.32 \\
\hline 13 & My family does not really listen when I talk about my cancer. & .51 & 1.57 & 1.25 & 1.42 & 0.98 \\
\hline 14 & I avoid talking about cancer to my family because I don't want to upset them. & .74 & 2.08 & 1.37 & 1.83 & 1.22 \\
\hline 15 & I don't tell my family about my problems because there is nothing they can do to help. & .74 & 1.95 & 1.29 & 2.17 & 1.40 \\
\hline 16 & I am frustrated when my family is overprotective of me because of my cancer. & .66 & 2.56 & 1.47 & 2.40 & 1.47 \\
\hline \multirow[t]{2}{*}{ Scale 2} & $\begin{array}{l}\text { Limitation of treatment } \\
\text { Cronbach's } \alpha=.51\end{array}$ & Factor loading & Mean & $\mathrm{SD}$ & Mean & SD \\
\hline & Explained variance, $12.52 \%$ & - & 3.26 & 1.18 & 2.52 & 1.06 \\
\hline 5 & If treatment caused financial hardship for my family, I would not take it. & .63 & 3.01 & 1.75 & 1.53 & 1.11 \\
\hline 7 & If treatment made me sick every day, I would not take it. & .79 & 2.82 & 1.57 & 2.85 & 1.54 \\
\hline 8 & $\begin{array}{l}\text { I could see that there could come a point when taking treatment would not } \\
\text { be worth the discomfort it causes. }\end{array}$ & .46 & 3.96 & 1.72 & 3.22 & 1.79 \\
\hline \multirow[t]{2}{*}{ Scale 3} & $\begin{array}{l}\text { Family involvement in treatment decisions } \\
\text { Cronbach's } \alpha=.68\end{array}$ & Factor loading & Mean & $\mathrm{SD}$ & Mean & SD \\
\hline & Explained variance, $12.06 \%$ & - & 4.90 & 1.18 & 4.53 & 1.33 \\
\hline 1 & My family plays a big role in the decisions I make about my cancer treatment. & .62 & 5.03 & 1.38 & 4.30 & 1.79 \\
\hline 10 & I value my family's judgement about treatment decisions. & .75 & 4.77 & 1.34 & 2.38 & 1.66 \\
\hline \multirow[t]{2}{*}{ Scale 4} & $\begin{array}{l}\text { Continuing treatment } \\
\text { Cronbach's } \alpha=.51\end{array}$ & Factor loading & Mean & $\mathrm{SD}$ & Mean & SD \\
\hline & Explained variance, $11.04 \%$ & - & 4.33 & 1.21 & 4.44 & 1.23 \\
\hline 3 & $\begin{array}{l}\text { In general, side effects are not really important when I consider my larger } \\
\text { goals of treatment. }\end{array}$ & .59 & 4.05 & 1.49 & 3.85 & 1.61 \\
\hline 4 & $\begin{array}{l}\text { Medical science may find a cure for cancer so I am willing to take any } \\
\text { treatment now to stay alive. }\end{array}$ & .67 & 4.59 & 1.45 & 5.00 & 1.42 \\
\hline \multicolumn{7}{|c|}{ Total explained variance, $56.66 \%$} \\
\hline & \multirow[t]{2}{*}{ Items without clear factor loading/excluded items } & & Mean & $\mathrm{SD}$ & Mean & SD \\
\hline & & & - & - & - & - \\
\hline 6 & My family and I have different views about the goal of treatment. & & 1.53 & 1.12 & 1.70 & 1.29 \\
\hline 9 & $\begin{array}{l}\text { I am willing to take treatment that causes me a significant amount of pain } \\
\text { if I can live a few months longer. }\end{array}$ & & 2.83 & 1.67 & 2.35 & 1.49 \\
\hline 11 & $\begin{array}{l}\text { My family's acceptance of my treatment decisions depends on how much } \\
\text { they like my doctor(s). }\end{array}$ & & 2.24 & 1.56 & 2.38 & 1.66 \\
\hline 12 & $\begin{array}{l}\text { It is important to base decisions about my cancer treatment on sources of } \\
\text { information other than my doctor. }\end{array}$ & & 2.48 & 1.56 & 2.92 & 1.64 \\
\hline 17 & $\begin{array}{l}\text { My family blames my cancer on my not having taken better care of myself. } \\
\text { [excluded due to extreme skewness] }\end{array}$ & & 1.29 & 0.73 & 1.39 & 0.94 \\
\hline 18 & $\begin{array}{l}\text { I would feel uncomfortable if the doctor began to talk to me about hospice } \\
\text { care. [excluded a priori] }\end{array}$ & & - & - & - & - \\
\hline
\end{tabular}


the CCAT-F) of item 17 ("My family blames my cancer on my not having taken better care of myself.") for patient and caregiver ratings led to its exclusion from further evaluation. First, CCAT-PF as patient/caregiver discrepancy score was computed according to the original scoring instructions (see Table 1) [4]. However, correlation of CCAT-PF discrepancy score did not yield any correlation with other theoretically related measures applied in the study. Assuming different subdomains of familial cancer-related communication, analysis continued with investigating the discrepancy scores between CCAT-P and CCAT-F by factor analysis. Scree plot and Kaiser-Guttman criteria suggested a five-factor solution. However, the fifth factor, covering items 13 and 14, was dropped due to low-content validity and very poor reliability $(\alpha=.31)$. In addition, items 15 and 16 were excluded, lacking distinct loadings on a factor. The final model led to four scales that were named 'Disclosure', 'Limitation of treatment,' 'Family involvement in treatment decisions,' and 'Continuing treatment' (see Table 1). These scales explained
$56 \%$ of the total variance with substantial factor loadings $>.45$ throughout.

Assessment of reliability and validity Scales demonstrated Cronbach's alphas in the range $\alpha=.51-.68$ (see Tables 1 and 2). Regarding construct validity, the Disclosure scale (patient version), describing poor cancer-related communication of the patient, was correlated with patient's distress (QSC: $r=.30 ; p<.0001$ ), unmet needs in several areas (SCNS-SF-34: $r=.25-.32 ; p<.001$ ), and negatively with social/family well-being (FACT: $r=-0.31 ; p<.0001$ ). In contrast, the latter was positively associated with Family involvement in treatment decisions and Continuing treatment. For caregivers, correlations for the Disclosure scale (caregiver version) were more pronounced with regard to anxiety (GAD-2: $r=.32 ; p<.0001)$ and psychological needs (SCNS-P\&C-G: $r=.35 ; p<.0001$ ). A lack of open communication from the caregiver's perspective was also associated with higher disease-related distress (QSC-R10: $r=.32 ; p<.0001)$ and strain (CSI: $r=.29 ; p<.0001)$ on the caregiver's side.

Table 2 Pearson correlation coefficients for CCAT-P and CCAT-F scales and related constructs

\begin{tabular}{|c|c|c|c|c|}
\hline & Disclosure & $\begin{array}{l}\text { Limitation } \\
\text { of treatment }\end{array}$ & $\begin{array}{l}\text { Family involvement } \\
\text { in treatment decisions }\end{array}$ & $\begin{array}{l}\text { Continuing } \\
\text { treatment }\end{array}$ \\
\hline \multicolumn{5}{|l|}{ Patient (CCAT-P) } \\
\hline SCNS_Psychological & $0.29 *$ & 0.21 & -0.10 & -0.07 \\
\hline SCNS - Heath system and information & $0.32 * *$ & 0.09 & -0.05 & -0.11 \\
\hline SCNS_-Physical and daily living & 0.21 & 0.16 & -0.06 & -0.16 \\
\hline SCNS_-Patient care and support & $0.26^{*}$ & 0.03 & -0.13 & -0.15 \\
\hline SCNS-Sexuality & $0.25 *$ & 0.17 & -0.14 & -0.14 \\
\hline PHQ-2 & 0.09 & 0.11 & -0.07 & -0.09 \\
\hline GAD-2 & 0.18 & 0.13 & -0.04 & -0.09 \\
\hline QSC-R10 & $0.30 * *$ & 0.21 & -0.18 & -0.23 \\
\hline FACT_Physical well-being & -0.12 & -0.08 & 0.07 & 0.22 \\
\hline FACT_Social/Family well-being & $-0.31 * *$ & -0.06 & $0.26^{*}$ & $0.27 *$ \\
\hline FACT_Emotional well-being & -0.16 & -0.20 & 0.13 & 0.14 \\
\hline FACT_Functional well-being & -0.13 & -0.11 & 0.12 & $0.29 * *$ \\
\hline FACT-Total & -0.13 & -0.10 & 0.11 & $0.28 * *$ \\
\hline \multicolumn{5}{|l|}{ Caregiver (CCAT-F) } \\
\hline SCNS-P\&C_-Psychological and emotional & $0.35^{* *}$ & 0.10 & -0.21 & -0.12 \\
\hline SCNS-P\&C-Health care service & 0.22 & 0.21 & -0.21 & -0.15 \\
\hline SCNS-P\&C_-Work and social needs & $0.30 * *$ & 0.20 & -0.09 & -0.09 \\
\hline SCNS-P\&C - Communication and family & $0.26^{*}$ & 0.11 & -0.18 & -0.09 \\
\hline PHQ-2 & -0.01 & -0.01 & -0.05 & -0.13 \\
\hline GAD-2 & $0.32 * *$ & 0.10 & -0.18 & -0.15 \\
\hline QSC-R10 & $0.32 * *$ & 0.13 & -0.12 & -0.18 \\
\hline CSI & $0.29 * *$ & 0.20 & -0.07 & -0.15 \\
\hline
\end{tabular}

${ }^{*} p<.001 ; * * p<.0001$ 
Subgroup analysis Subgroup analyses were conducted for gender, age group, caregiver type, tumor site, and WHOECOG performance status. Spouses of patients scored higher on Continuing treatment than other family members ( $\Delta$ mean [SD], 0.63 [1.22]; $p<.05)$. Patients scored significantly higher on Family involvement in treatment decisions if either they $(\Delta$ mean [SD], $0.75[1.13] ; p<.0001)$ or their caregiver $(\Delta$ mean [SD], $0.47[1.16] ; p<.01)$ were aged 60 years or older.

\section{Discussion}

This study aimed for a deeper insight in conflictual communication patterns of cancer patients and their families. We therefore undertook a psychometric reassessment of the CCAT-PF using a German version with special regard to evaluation of the scale structure. Whereas the original score covers a wide range of content areas where families might disagree, we aimed for higher content validity in this study using subdomains of CCAT-PF separately. Conceptualizing communication as a multidimensional construct, our exploratory factor analysis revealed a fourfactor model with scales demonstrating sufficient internal consistencies to allow for content-specific interpretation. Higher social quality of life in the patient-caregiver-relationship went along with the wish to continue treatment besides low chances and/or high side effects. In this case, greater family involvement in decisions regarding treatment was also observed. When considering disclosure, a lack of dialogue was associated with higher distress and consequently higher needs by both parties, but for the caregiver, it also entailed a significantly higher degree of anxiety and strain. We could not confirm a significant association between communication and depression, as previously reported [4]. Overall, the Disclosure scale originating from the CCAT-PF emerged as a short, valid, and reliable tool to assess risks for impaired affective adjustment in patients and caregivers dealing with cancer. Higher scores on the scale as an indicator for low cancer-related communication were significantly associated with considerable decrements in emotional and social wellbeing for caregivers; this association was more pronounced in caregivers than for patients. This observation is in accordance with the notion that caregivers especially might be negatively affected when patients choose to withhold communication [15-17]. In accordance with the original work [4] emphasizing the necessity of separate examination of individual domain scores, in addition to the CCATPF total score, we propose the Disclosure subscale as a stand-alone instrument for identifying patient-caregiverdyads at risk. Tailoring psychosocial interventions to improve open communication may help patients and their caregivers better adjust to a cancer diagnosis [18].

Conflict of interest In accordance with the Recommendations for the Conduct, Reporting, Editing, and Publication of Scholarly Work in Medical Journals proposed by the International Committee of Medical Journal Editors (ICMJE), the authors' declare that there is no conflict of interest. The study was conducted without involvement of a sponsor.

The authors had full access to all of the data in this study and take complete responsibility for the integrity of the data and the accuracy of the data analysis.

\section{References}

1. Lewis FM (2009) The family's "stuck points" in adjusting to cancer. In: Holland JC, Breitbart WS, Jacobsen PB (eds) Psycho-Oncology. Oxford University, New York, pp 511-515

2. Manne SL et al (1999) Spousal negative responses to cancer patients: the role of social restriction, spouse mood, and relationship satisfaction. J Consult Clin Psychol 67(3):352-361

3. Burns CM et al (2007) Fluctuating awareness of treatment goals among patients and their caregivers: a longitudinal study of a dynamic process. Support Care Cancer 15(2):187-196

4. Siminoff LA et al (2008) The Cancer Communication Assessment Tool for Patients and Families (CCAT-PF): a new measure. Psychooncology 17(12):1216-1224

5. Siminoff LA et al (2006) Measuring discord in treatment decisionmaking; progress toward development of a cancer communication and decision-making assessment tool. Psychooncology 15:528-540

6. Bonett DG (2002) Sample size requirements for testing and estimating coefficient alpha. J Educ Behav Stat 27(4):335-340

7. Escobar Pinzon LC et al (2010) End-of-life care in Germany: study design, methods and first results of the EPACS study (Establishment of Hospice and Palliative Care Services in Germany). BMC Palliat Care 9:16

8. Book K et al (2011) Distress screening in oncology_evaluation of the Questionnaire on Distress in Cancer Patients-Short Form (QSCR10) in a German Sample. Psychooncology 20(3):287-293

9. Kroenke $\mathrm{K}$ et al (2009) An ultra-brief screening scale for anxiety and depression: the PHQ-4. Psychosomatics 50(6):613-621

10. Girgis A, Lambert S, Lecathelinais C (2011) The supportive care needs survey for partners and caregivers of cancer survivors: development and psychometric evaluation. Psychooncology 20(4):387393

11. Cella DF et al (1993) The Functional Assessment of Cancer Therapy scale: development and validation of the general measure. J Clin Oncol 11(3):570-579

12. Cella DF et al (1995) Reliability and validity of the Functional Assessment of Cancer Therapy-Lung (FACT-L) quality of life instrument. Lung Cancer 12(3):199-220

13. Robinson BC (1983) Validation of a Caregiver Strain Index. J Gerontol 38(3):344-348

14. Nunnally JC, Bernstein IH (1994) Psychometric theory, 3rd edn. McGraw-Hill, New York

15. Fife BL et al (2013) Partner interdependence and coping with lifethreatening illness: the impact on dyadic adjustment. J Fam Psychol 27(5):702-711

16. Kornblith $\mathrm{AB}$ et al (2006) Cancer-related communication between female patients and male partners scale: a pilot study. Psychooncology 15(9):780-794 
17. Lo C et al (2013) Couples facing advanced cancer: examination of an interdependent relational system. Psychooncology 22(10):22832290
18. Northouse LL et al (2013) Randomized clinical trial of a brief and extensive dyadic intervention for advanced cancer patients and their family caregivers. Psychooncology 22(3):555-563 